\title{
tic\&société
}

Vol. $4, n^{\circ} 1 \mid 2010$

Interactivité et lien social

\section{De la communauté à l'écume : quels concepts de sociabilité pour le « web social »?}

Bernhard Rieder

\section{CpenEdition}

Journals

Édition électronique

URL : http://journals.openedition.org/ticetsociete/822

DOI : $10.4000 /$ ticetsociete. 822

Éditeur

Association ARTIC

\section{Référence électronique}

Bernhard Rieder, «De la communauté à l'écume : quels concepts de sociabilité pour le « web

social »? », tic\&société [En ligne], Vol. 4, n 1 | 2010, mis en ligne le 17 mai 2010, consulté le 14 novembre 2019. URL : http://journals.openedition.org/ticetsociete/822 ; DOI : 10.4000/ticetsociete. 822 
tic\&société - 4 (1), 2010

\title{
De la communauté à l'écume: quels concepts de sociabilité pour le « web social»?
}

\author{
Bernhard Rieder \\ Maître de conférences \\ Université de Paris VIII \\ Laboratoire Paragraphe \\ 16 rue de Rouen \\ F - 75019 Paris \\ bernhard.rieder@univ-paris8.fr
}

Bernhard Rieder est maître de conférences au département hypermédia de l'université Paris 8 et membre du laboratoire paragraphe. Travaillant comme développeur Web à partir du milieu des années 90 il soutient une thèse en Sciences de l'information et de la communication en 2006. Son travail porte principalement sur le rapport entre technique et société, la médiation des pratiques sociales et la production de connaissances par les outils numériques et la question de la conception des systèmes informatiques comme pratique politique. 
De la communauté à l'écume : quels concepts de sociabilité pour le « web social »?

\title{
De la communauté à l'écume : quels concepts de sociabilité pour le « web social » $?^{1}$
}

\begin{abstract}
Résumé
Dans cet article nous proposons un examen du « web social » sous l'angle des rapports entre applications interactives en ligne et formes de sociabilité. Une typologie des systèmes composant le web social est présentée. Nous analysons trois concepts de sociabilité - communauté, réseau et foule - et introduisons un quatrième concept, l' « écume » de Peter Sloterdijk, pour élucider des rapports sociaux éphémères et peu stables qui prolifèrent sur l'internet. Nous montrons comment le triplet écume - bulle - membrane peut rendre compte des nouvelles configurations sociotechniques, caractérisées par un individualisme de masse et oscillant entre relation et isolation.
\end{abstract}

Mots-clés : web social, sociabilité, technique et société, écume, épistémologie

\begin{abstract}
In this paper we propose to examine the "social Web" according to the relations between interactive online applications and forms of sociability. A typology of the systems that make up the social Web is put forward. We analyze three concepts of sociability - community, network, and crowd - and introduce a fourth one, Peter Sloterdijk's "foam", to take get grip on the ephemeral and instable relations that proliferate on the Internet. We show how the triplet foam - bubble - membrane can shed light on the new sociotechnical configurations, which are characterized by mass-individuality, oscillating between relation and isolation.
\end{abstract}

Keywords: social Web, sociability, technology and society, foam, epistemology

\section{Resumen}

En este artículo se propone un examen de la "web social" desde el punto de vista de las relaciones entre aplicaciones interactivas en línea y las formas

\footnotetext{
${ }^{1}$ Une première version de ce texte à été présentée, sous le titre "Membranes numériques : des réseaux aux écumes » dans le séminaire PHITECO à l'Université Technique de Compiègne le 29 janvier 2008.
} 


\title{
Bernhard RIEDER
}

\begin{abstract}
de sociabilidad. Se presenta una tipología de los sistemas que componen la web social. Se analizan tres conceptos de sociabilidad -comunidad, red y muchedumbre- y se introduce un cuarto concepto: la "espuma" de Peter Sloterdijk, para explicar las relaciones sociales efímeras e inestables que proliferan en Internet. También se muestra cómo la tripleta espuma-burbujamembrana puede dar cuenta de las nuevas configuraciones sociotechniques, que se caracterizan por un individualismo de masas, que oscila entre aislamiento y relación.
\end{abstract}

Palabras claves: web social, sociabilidad, técnica y sociedad, espuma, epistemología 
De la communauté à l'écume : quels concepts de sociabilité pour le « web social »?

Pendant longtemps, les outils et machines ont été pensés comme faisant partie d'une catégorie comprise comme essentielle, la technique, dont les qualités pourraient peut-être varier sur le plan du phénotype - les objets affichent visiblement des formes et fonctions fort différentes - mais découleraient en fin de compte d'un même génotype, la tekhnè, la façon de faire efficace. Pour connaître le rôle que jouent les objets techniques dans et pour la société, il suffirait de comprendre la technique, de manière abstraite - ce qui dispense, fort à propos, de regarder de plus près, d'ouvrir la boîte noire. Or, depuis que Simondon a déclaré la différence entre diode et triode digne de réflexion philosophique, l'école essentialiste a perdu du terrain et les différents déterminismes reculent au profit de perspectives théoriques et méthodes empiriques qui conçoivent la technique comme hétérogène, de natures différentes. Alors qu'elle est pensée comme multiple, ses « effets » - si l'on veut entretenir l'image d'une causalité simple - peuvent donc varier abondamment et les différences entre objets, sur le plan des formes et fonctions, cessent d'être négligeables pour l'analyse. En même temps, un tel changement de perspective ne rend point invalide la question de la relation entre technique(s) et société(s) ; mais le doute sur nos a priori nous force de la poser autrement, de manière plus nuancée et surtout en prenant en compte l'immense complexité qui marque aujourd'hui les deux composants de cette relation.

Dans cet article, nous nous proposons d'étudier, sur le plan conceptuel, un phénomène relativement récent dont l'un des noms courants, le " web social », nous jette au cœur du problème: il s'agirait d'une structure technique particulière qui serait non seulement dans la société comme tout autre artefact utilisé par les êtres humains mais en quelque sorte lieu de société. Mais est-ce qu'il ne s'agit pas d'une tautologie? Lorsqu'on admet que tout dispositif de communication peut jouer un rôle dans la création et le maintien de liens sociaux, cette combinaison de termes paraît étrange. Or, si nous prenons ce terme « web social » 2 comme point de départ d'une interrogation, nous pouvons légitimement nous demander pourquoi ce web là serait plus social que les autres et en quoi consisterait précisément sa "socialité ». Notre propos est donc d'examiner comment un ensemble d'applications informatiques accessibles à travers un réseau peuvent être compris comme artefact technique, et plus particulièrement à travers sa capacité à permettre à des individus de se rassembler dans des constellations parfois surprenantes. Cet

${ }^{2}$ Cette notion est souvent mentionnée en liaison avec - où simplement comme synonyme de - celle de «Web 2.0 ». Loin d'être des vocables scientifiques, ces deux termes ont surtout joué un rôle intéressant dans la renaissance de l'économie de l'internet après l'éclatement de la bulle spéculative en $2001 / 2002$. 
artefact mérite que l'on prête attention à sa technicité et, en guise d'introduction, il nous semble important de commencer avec deux observations pour établir le fond de sa particularité.

Premièrement, l'internet repose sur une base technique malléable. L'ordinateur est une machine universelle qui sait se transformer selon le programme qu'elle exécute - le champ des formes et fonctions que l'on peut concevoir et implémenter est donc par principe illimité. (Turing, 1948) De manière analogue, les protocoles qui règlent la circulation des paquets sur l'internet fonctionnent, au moins pour le moment, conformément au principe du end-to-end (Saltzer, Reed et Clark, 1984), selon lequel les services proposés ne sont pas définis par l'architecture du réseau mais par les parties communicantes, c.-à-d. les ordinateurs qui échangent des données. Par conséquent, pour ajouter une nouvelle fonctionnalité à l'internet, il suffit de distribuer le logiciel qui l'implémente aux internautes, aucune adaptation de l'infrastructure n'étant nécessaire. ${ }^{3}$ Sur le web - lui même une innovation logicielle - cette logique est poussée à l'extrême : les interfaces des sites se déploient à travers le navigateur et le seul véritable obstacle à la diffusion d'une nouvelle application est à trouver dans les méandres de l'économie de l'attention (Goldhaber, 1997). De nouvelles fonctionnalités, activités et contenus sont proposés tous les jours et malgré les conventions et tendances qui le structurent, l'espace de possibilités est immense.

Deuxièmement, grâce à cette " plasticité » l'internet a pu devenir un médium multiforme qui peut accueillir différentes configurations communicationnelles. II faut rappeler, avec Flichy, que "parmi les nombreuses technologies de communication inventées au cours de ce siècle, aucune n'est adaptée à la communication de groupe " (Flichy, 1999, p.94); l'internet rompe avec cette tradition et semble réaliser les espérances que certains intellectuels engagés (Benjamin, Brecht, Enzensberger, etc.) avaient pu formuler par rapport à d'autres média, à savoir de mettre en place un moyen de communication " de nombreux à nombreux" (many-to-many). Nous savons maintenant que cela n'abolit pas le pouvoir - et par ailleurs non plus la monopolisation et la centralisation - mais il paraît évident que les régimes de communication subissent une transformation profonde dont l'un des éléments est un potentiel inédit de contournement des gatekeepers traditionnels et de dépassement des limites structurelles des média de masse.

\footnotetext{
${ }^{3}$ Le logiciel Skype p.ex. permet d'utiliser son ordinateur comme téléphone, Joost le transforme en téléviseur; il a certes fallu attendre une certaine bande passante pour rendre ces applications viables mais elles font usage, pour l'instant, du même protocole IPv4 introduit en 1981.
} 
De la communauté à l'écume : quels concepts de sociabilité pour le « web social »?

Dans cette première analyse, le terme " web social » évoque une matérialité technique malléable et diversifiée dont l'une des capacités consiste à pouvoir médiatiser la communication - et d'autres interactions - de (très) grand groupe. Avant de passer à la question des formes de sociabilité, il convient de spécifier le phénomène en plus de détail.

\section{Le Web social}

L'internet propose des outils d'interaction de groupe bien avant qu'on ait entendu parler d'un "web social ». Les newsgroups, mailinglists, forums et chats commencent à fleurir dans les années quatre-vingt et le web lui-même fût conçu pour faciliter l'échange. De notre point de vue, les applications que l'on regroupe habituellement derrière le terme " web social » se distinguent de leurs prédécesseurs parce qu'elles vont plus loin dans l'organisation et la structuration des interactions entre usagers que les outils " historiques "; elles se spécialisent dans des activités particulières et proposent des interfaces et fonctionnalités optimisées pour les tâches en question. Un site comme YouTube n'est pas simplement un site d'échange mais un site d'échange de vidéos et toute la plate-forme est organisée autour de ce type de contenu. On pourrait facilement utiliser un newsgroup pour échanger et discuter des vidéos (et ce type de newsgroup existe effectivement) mais un système spécialisé facilite énormément tous les aspects de l'activité, du téléchargement vers le serveur à la navigation dans les archives. Pour une communauté qui serait prête à négocier, appliquer, et (faire) respecter les normes et conventions (techniques aussi bien que sociales) nécessaires à la transformation d'un newsgroup en club vidéo, une très grande discipline s'imposerait. Sur YouTube, une partie de ce travail de standardisation et d'organisation sociale est assurée par la plateforme technique; elle est déléguée sur un ensemble d'interfaces et d'algorithmes (Rieder 2006). De manière analogue, certains éléments significatifs de la création et du maintien de sociabilité sont formalisés en forme de code informatique, notamment le réseautage social et la gestion de l'attribution de qualité. Les fonctionnalités omniprésentes pour créer et gérer des listes d' " amis " et pour "voter" sur les contenus ou commentaires rendent moins ambigües et simplifient des pratiques sociales et les adaptent à la vitesse ambiante. II s'agit d'" architectures discursives ॥ (Sack, 2005), des systèmes qui définissent de manière relativement détaillée les modalités d'interaction et de communication qu'ils intègrent. Le succès du web social peut être attribué, au moins partiellement, à cette pré-structuration autour d'activités particulières, qui canalise les possibilités d'action tout en gardant l'espace de possibilités suffisamment ouvert pour permettre une appropriation riche du système par les usagers. 
Afin de rendre notre analyse plus claire, nous proposons une classification des plates-formes autour de quatre activités: publier, organiser, socialiser et rencontrer. Pour illustrer le propos, qui nous sera bien utile plus bas, nous rattachons chaque activité à un type d'application emblématique.

- Publier: Les blogs sont devenus les principaux vecteurs d'un phénomène de publication de masse. Leurs interfaces simples ont réussi à baisser considérablement le seuil à franchir pour qu'un internaute puisse devenir émetteur. De plus, les différentes méthodes pour connaître les sites qui référencent un billet de blog (backlinking) ont pour effet de donner lieu à de réseaux conversationnels denses qui forment des structures communicationnelles interconnectées, les blogosphères.

- Organiser: Les plates-formes de gestion et de partage d'information constituent une deuxième catégorie. Des systèmes comme delicious, CiteUlike, Connotea, StumbleUpon ou Furl proposent à leurs utilisateurs d'organiser et d'échanger leur collection de liens, Flickr offre un service de stockage et partage pour les photos et YouTube et Dailymotion pour la vidéo. Ces sites s'organisent typiquement autour d'un système de "classification de masse " (folksonomy) où chaque objet est décrit par un ou plusieurs mots-clé (tags). Le vocabulaire émergeant sert à produire des chemins de navigation et à chercher dans l'ensemble des contenus.

- Socialiser: Les sites de réseautage social (MySpace, Facebook, Friendster, hi5, Bebo, Linkedln, etc.) sont structurés autour la double notion de profil d'utilisateur et de carnet de contacts. Les utilisateurs précisent leurs goûts, valeurs, choix, préférences ou compétences, ils forment ou soignent des liens sociaux et il interagissent, à travers différents moyens de communication et d'échange, avec d'autres membres du système.

- Rencontrer: Les sites de rencontre ressemblent en partie aux sites de réseautage. Ils ne servent pourtant pas nécessairement à la création et au maintien d'un réseau de contacts, mais avant tout à la recherche d'un compagnon ou partenaire sexuel. L'accent est donc mis sur la facilitation de la prise de contact avec d'autres personnes.

La plasticité du substrat technique fait que ces catégories ont tendance à s'hybridiser dans la plupart des systèmes actuels. II s'agit donc plutôt d'une liste (non exhaustive) d'activités que de véritables catégories de systèmes. Publication, organisation, réseautage social et rencontre; un site comme Facebook peut effectivement intégrer tous les quatre. 
De la communauté à l'écume : quels concepts de sociabilité pour le « web social »?

Bien évidemment, les services mentionnés flottent sous la bannière du « web social » - ou alors de "web 2.0 », notion encore plus médiatisée - et ces dernières années des milliers d'articles les ont décrits et analysés. Une certaine familiarité avec les objets en question peut donc être présupposée. De ce fait, nous allons nous concentrer sur une problématique particulière qui nous paraît encore relativement peu développée. Les recherches autour du "web 2.0 " traitent de préférence la question de la participation et cherchent à théoriser la production de messages et d'objets par des personnes historiquement reléguées à la place de purs consommateurs ${ }^{4}$ ou spectateurs ${ }^{5}$. Derrière ce phénomène de contournement des canaux de distribution traditionnels se cache une question plus élusive, celle de savoir quelles figures de sociabilité se développent dans les « assemblages d'internautes » qui se constituent à travers ces nouvelles pratiques de communication et de partage. Au delà de l'évolution des rapports entre production et consommation, quelles sont les formes d'être ensemble qui émergent dans les espaces générés par les systèmes dont se composerait un web social ? La réponse que l'on peut entendre souvent, selon laquelle ils seraient simplement des communautés d'usagers qui discutent et créent, ne nous semble guère en accord avec l'hétérogénéité des configurations que l'on peut observer empiriquement. Pour préparer des analyses plus fines, nous allons donc rapidement revoir les trois notions de morphologie sociale les plus courantes avant d'en proposer une quatrième qui nous paraît particulièrement apte à mettre en rapport lien social et machine interactive.

\section{Communauté, réseau, foule}

Comment parler des groupes ? Quels termes utiliser ? Les sciences sociales nous offrent bien évidemment un grand nombre de concepts pour penser les différentes formes d'être ensemble. Trois notions nous paraissent dominantes dans les recherches sur la sociabilité en ligne: la communauté, le réseau et la foule.

\subsection{Communauté}

Le terme "communauté " est habituellement associé aux travaux de Ferdinand Tönnies (Tönnies, 1887) qui fait la distinction entre Gemeinschaft (communauté), une association d'êtres humains où le groupe est prioritaire par

\footnotetext{
${ }^{4}$ Des termes comme "ProAm» (Leadbeater et Miller, 2004) ou «Produsage » (Bruns, 2008) cherchent à témoigner de la porosité de la frontière entre producteur et consommateur.

5 Jenkins (Jenkins, 2006) parle ainsi d'une audience " interactive » dont l'activité ne se résume pas à l'interprétation des messages - démontrée par Barthes et les Cultural Studies - mais inclue leur production.
} 
rapport à l'individu, et Gesellschaft (société) où l'intérêt particulier emporte sur l'intérêt commun. Les concepts durkheimiens de solidarité mécanique et solidarité organique forment une dichotomie assez proche. La communauté implique donc un commun - des idées, valeurs et signes partagés qui établissent la particularité du groupe; l'identité des membres du groupe est alimentée par cette identité collective. Latham et Sassen argumentent qu'une communauté consiste en "a common identity around shared goals and reciprocal relations among participants, and that such identity, goals, and reciprocity are an important and substantive aspect of each of participant's life, professional or personal » (Latham et Sassen, 2005, p. 12). Le fameux livre de Howard Rheingold (Rheingold, 1993), qui adapte le concept au numérique en introduisant le terme de "communauté virtuelle », décrit une plate-forme en ligne - le WELL - dont les membres cherchaient effectivement à établir une telle communauté à travers câbles et écrans. Certes, le WELL n'accueillait pas un groupe complètement homogène mais nombre d'éléments indiquent qu'il hébergeait une communauté dans le sens de la définition donnée plus haut : les membres passaient un temps important dans le système ; ils se connaissaient bien; leurs relations s'inscrivaient dans la durée; la conversation était réciproque et circulaire ; certaines personnes forgeaient des rapports importants hors ligne; les questions de gouvernance du système stimulaient une vive participation; le groupe visait le consensus par discussion; les relations jouaient une rôle important dans la vie privée et la vie professionnelle de ses membres; le groupe partageait certaines valeurs (démocratie directe, libéralisme ou libertarisme, optimisme technologique, etc.), une étiquette et une identité commune de pionnier social et technique. Bien évidemment, très peu des groupes que l'on peut observer en ligne affichent un tel degré d'intégration et c'est rarement le cas dans les plates-formes du web social qui accueillent souvent des publics très hétérogènes dont la majorité des relations sont faibles, éphémères et dispersées. L'émergence d'une véritable identité commune est de loin l'exception et non la règle. En même temps, l'usage du terme prolifère : les sites proposant des services "sociaux" et les médias qui s'en font l'écho parlent d'une "communauté Facebook » ou d'une "communauté MySpace " en impliquant que le fait d'être inscrit sur un même système constitue une réalité partagée suffisamment forte pour conférer le statut de communauté.

Au-delà de son aspect de marketing, ce choix reflète un honnête désir, lié à la religion civile américaine, de faire fonctionner l'idéal démocratique communautaire $^{6}$ à l'échelle de millions de personnes. D'un point de vue

\footnotetext{
${ }^{6}$ La démocratie américaine se fonde sur des principes bien différents que la république française. L'idéale communautaire, rural et méfiant des hiérarchies, est encore aujourd'hui incarné par le
} 
De la communauté à l'écume : quels concepts de sociabilité pour le « web social »?

scientifique, un tel usage n'a qu'une faible valeur heuristique. En réalité, la communauté virtuelle, dans le sens donné en haut, est quelque chose de rare.

\subsection{Réseau}

Le deuxième concept, celui de réseau, connaît actuellement une immense popularité aussi bien dans les disciplines scientifiques que dans le discours populaire. La modélisation par nœuds et connections paraît particulièrement bien adaptée à nos sociétés flexibles, progressivement déhiérarchisées et hautement technisées. Le réseau est un concept suffisamment abstrait - une qualité qu'il partage avec d'autre «métaconcepts» comme système ou structure - pour l'appliquer à des phénomènes très variés. Autour de l'analyse des réseaux sociaux (Degenne et Forsé, 2004), une branche de la sociologie, et d'une "science des réseaux" (Watts, 2005) en cours de formation, des méthodologies et vocabulaires puissants ont été développés. L'étude de la morphologie sociale a fait un grand pas en avant, entre autre grâce à un usage fécond de la théorie des graphes, qui permet des mesurer certaines caractéristiques d'un réseau comme sa densité, connexité, réciprocité ou distribution de degré. Ces approches sont souvent appliquées empiriquement aux relations sociales sur l'internet où les traces laissées par les échanges des internautes constituent de formidables corpus à explorer. Le concept de réseau recèle pourtant deux problèmes qui l'empêchent d'étendre sa prépondérance dans les modes d'appréhension des phénomènes sociaux observables sur l'internet: premièrement, à cause de son degré d'abstraction élevé et de sa perspective surtout topologique il ne peut que difficilement rendre compte des questions de qualité, notamment celles ayant attrait à la signification du caractère des relations et à l'identité des nœuds ; deuxièmement, les concepts actuels ont du mal à exprimer les relations éphémères et les dynamiques variables. Bien que certainement plus flexible que la définition forte de communauté, le concept de réseau implique encore des relations plutôt stables, plutôt cohérentes et nécessairement " traçables "; il ne rend guère compte des interactions subtiles et indirectes qui caractérisent une importante partie de la sociabilité du web social. Deux internautes qui taguent leurs photos avec le même mot-clé, des usagers qui commentent la même vidéo, une personne à la recherche d'un compagnon qui jette un regard rapide sur une photo pour tout de suite passer à la prochaine, ce sont des cas d'une proto-sociabilité qui passent en dessous du seuil de "relation" et même de "relation faible " (weak tie) (Granovetter, 1973). Finalement, le concept de réseau est difficilement

«New England Town Hall Meeting » une assemblée complète des citoyens d'une ville qui dispose d'un pouvoir décisionnel important. 
Bernhard RIEDER

applicable à la question de savoir comment ces contacts éphémères peuvent se transformer en quelque chose de plus stable.

\subsection{Foule}

Le troisième concept cherche effectivement à rendre compte des interactions de masse très peu stables. La notion de foule est historiquement liée à la naissance d'une culture urbaine de masse (Le Bon, 1895) et au fascisme (Canetti, 1960), bien que les connotations ne soient, aujourd'hui, plus exclusivement négatives. Certains aspects centraux du concept ont tout de même persisté. Le Bon écrit que " [d]ans certaines circonstances données [...] une agglomération d'hommes possède des caractères nouveaux fort différents de ceux de chaque individu qui la compose " (Le Bon, 1895, p. 9) et décrit ainsi quelque chose que nous qualifierons aujourd'hui de phénomène d'émergence. Lorsque certains parlent maintenant de la "sagesse des foules" (the wisdom of crowds) (Surowiecki, 2004) il s'agit bien de l'idée que l'agrégation de l'information qui circule dans un ensemble d'individus de faible cohésion peut donner lieu à des "caractères nouveaux », c.-à-d. à des résultats (sur les plans de la cognition, coordination et coopération) largement supérieurs aux performances d'un membre quelconque à lui seul. La "sagesse " est donc conçue en tant que propriété émergente du groupe en question. Certaines applications en ligne qui visent l'activité d'organisation, notamment les applications de "social news " (Slashdot, Digg, Newsvine, etc.) et les différents systèmes d'évaluation de produits, services ou personnes, essayent explicitement de produire et rendre opératoire un tel effet pour classer ou filtrer les objets qu'ils référencent. Les auteurs qui s'intéressent à ces phénomènes de foule se concentrent habituellement sur la problématique de la performance cognitive émergente - notamment par rapport aux performances de méthodes algorithmiques sans participation humaine - ainsi que sur la question plus abstraite d'une éventuelle "démocratisation » des processus de décision par l'agrégation de " micro-décisions ». Rares sont les réflexions plus approfondies sur la sociabilité que représentent les interactions indirectes qui se produisent au sein des populations participantes. En outre, il existe aujourd'hui des interactions de masse qui ne sont pas dirigées vers la performance cognitive ou la classification d'informations ; les échanges de références, photos, vidéos, commentaires, pokes ${ }^{7}$, liens, votes et pageviews qui prolifèrent sur le Web

7 Sur Facebook, la fonctionnalité "poke » qui permet de «pousser» quelqu'un représente en quelque sort le degré zéro de l'interaction. L'utilisateur poussé $(A)$ reçoit un message qu'une personne (B) lui a poussé, et c'est tout. Cette fonctionnalité était, au début, l'une des plus utilisées du système.

tic\&société - 4 (1), 2010 
De la communauté à l'écume : quels concepts de sociabilité pour le « web social »?

social ne se dirigent finalement que rarement vers des objectifs communs. Le concept de foule renvoie à une dissolution - réelle ou analytique - de l'individu

dans la masse comme acteur intégré mais lorsqu'on regarde les usages et pratiques des internautes, on se rend vite compte que leurs efforts sont, au contraire, majoritairement tournés vers l'individu et la production d'identité.

\subsection{Identité et réseau}

La complexité des rapports entre individu et société est au cœur de l'horizon d'investigation des sciences humaines et sociales; les réseaux interactifs s'introduisent dans ce rapport, y installent de nouveaux variables et déstabilisent notre appareil conceptuel. Selon Manuel Castells (Castells, 1996-1998), une tension entre identité et réseau caractérise notre condition actuelle et nous pensons effectivement que les pratiques qui émergent sur le web (social) ne peuvent pas être comprises à travers une perspective qui serait purement relationnelle, qui ne prendrait pas en compte la singularité et l'intériorité des unités en relation. Nos rapports aux autres sont en effet pris dans une logique vacillante entre l'ouverture vers l'autre et le repli sur soi ; la morphologie sociale qu'affiche une population donnée est définie par un jeu entre relation et isolation. Bien qu'ils ne puissent pas les déterminer, les architectures (techniques) de communication et d'interaction affectent la façon dont ce jeu se joue et, par conséquent, les structures sociales produites. Sur le web social se développent effectivement des façons particulières de gérer relation et isolation et nous arguons que les concepts classiques de communauté, réseau et foule ne suffisent pas pour rendre compte de la totalité des phénomènes de sociabilité qui en résultent. Chacune de ces notions conserve une capacité heuristique indéniable mais comme nous l'avons vu, le web favorise des formes d'être ensemble qui échappent à notre appareil conceptuel. Nous proposons donc une quatrième manière de concevoir cette sociabilité à faible stabilité ; il s'agit de la notion d'écume.

\section{Ecumes}

Écumes est le titre du troisième tome de la trilogie Sphères du philosophe allemand Peter Sloterdijk (Sloterdijk, 1998-2004) ; l'objectif de cet opus magnum consiste à repenser la condition humaine à travers les «espaces de coexistence " produits dans l'évolution des civilisations. La métaphore de l'écume y figure comme l'outil principal pour penser nos " sociétés d'individus " (Individualgesellschaft), conditionnées par le paradoxe de l'« individualisme de masse " (Massenindividualismus), où les "bulles » individuelles se retrouvent 
Bernhard RIEDER

en situation de co-isolation: elles composent un ensemble dense dans lequel elles sont toujours confrontées aux autres bulles et donc forcées, à cause des infiltrations provenant des autres, d'œuvrer en permanence pour la maintenance de leur cohérence interne. Chez Sloterdijk, la tension entre individu et société n'est pas résolue par un geste de synthèse, elle est conçue comme une double dynamique, constitutive de l'expérience sociale : dans l'écume il y a à la fois coisolation et co-fragilité. La bulle doit s'isoler des autres mais son extension intérieure (son identité ou "foyer de sens ») dépend de la production par ces autres d'un espace qui leur est « propre $» .^{8}$ Notre identité doit se produire par à la fois relation et isolation, ou - pour parler en termes bourdieusiens - le jeu de la distinction demande que tout le monde y joue. Nous ne pouvons pas ici développer plus avant notre vision du travail de Peter Sloterdijk, mais nous allons tout de même essayer de proposer quelques pistes de réflexion ouvertes par la métaphore d'écume.

\subsection{Ecumes numériques}

II faut d'abord remarquer que les Sphères de Sloterdijk ne font guère référence aux technologies numériques. Sur le plan des artefacts, il s'agit avant tout d'une considération de l'architecture et de l'urbanisme comme manifestations matérielles de la condition actuelle de nos sociétés. Cependant, la métaphore de l'écume peut être facilement développée en direction des systèmes et pratiques du web social. Nous proposons d'interpréter l'immense production de contenus de toute sorte par les internautes - de messages, images, vidéos, listes de préférences, auto-descriptions, aménagements de profils, systèmes de classification, etc. - comme un travail de création et de maintien d'une bulle, c'est-à-dire d'un espace propre, d'une identité, d'une extension de sens. Selon Danah Boyd (Boyd, 2006), les internautes « se font exister par l'écriture " (write themselves into being); et quand le théoricien néerlandais Geert Lovink (Lovink, 2006) parle des blogs comme signes d'un narcissisme rampant, il suggère que le style d'écriture qui caractérise la blogosphère, c'est-à-dire la prépondérance de l'opinion et du subjectif, est un signe que les blogs servent à remplir une fonction identitaire ${ }^{9}$ aussi bien qu'une fin communicationnelle. Un regard rapide sur Facebook ou MySpace peut donner une impression similaire : le travail investi dans la création et le soin des profils est souvent considérable - les bulles sont aménagées, décorées et

\footnotetext{
${ }^{8}$ Notons ici l'air de famille avec la philosophie dialogique de Martin Buber et, à moindre degré, d'Emmanuel Lévinas, selon laquelle l'autre est condition nécessaire pour l'existence d'un moi.

${ }^{9}$ Pour Lovink il s'agit effectivement d'une identité particulière, narcissique et incapable de distinguer entre les registres du privé et du public.
}

tic\&société - 4 (1), 2010 
De la communauté à l'écume : quels concepts de sociabilité pour le « web social »?

constamment mises à jour. Examiné de plus près, on voit que ces éléments " déclaratifs " sont complémentés par une "identité agissante " (Georges et al., 2009) qui peut impliquer la rédaction de billets, commentaires et notices, l'activité dans des groupes ou sur le profil d'autres utilisateurs, ainsi que d'autres gestes de "participation ». Cette mise en scène de soi demande naturellement une attention continue et ce travail est à la fois dirigé vers les autres et soimême. Certes, la répartition entre orientations de type «identité » ou « relations » peut varier d'une application à l'autre et d'individu à individu.
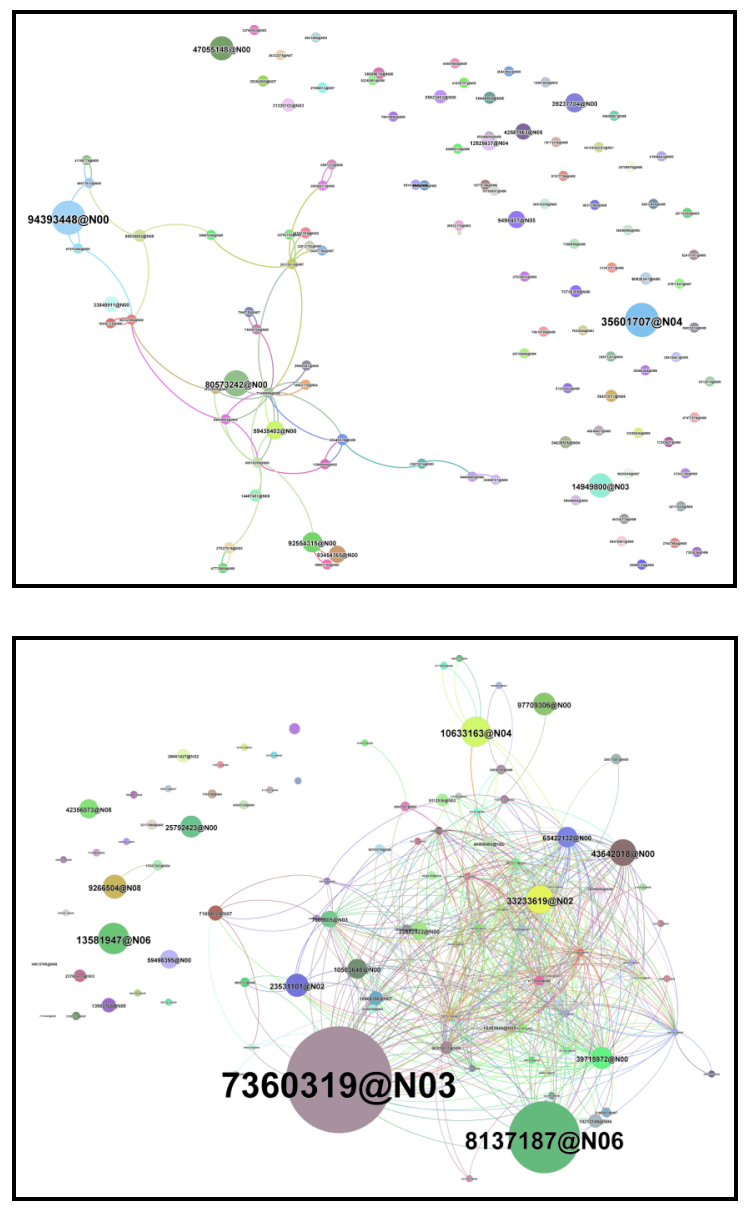

Fig. 1.: Les deux graphes (réalisés à partir de l'API de Flickr avec notre propre crawler et GEPHI comme logiciel de visualisation) montrent deux groupes sur Flickr 
(haut: "iPod love », bas : "Vienna @ Night»). Les nœuds représentent des utilisateurs ayant posté plus de cinq photos dans le groupe et les arcs les relations d'amitié entre eux. Bien que le nombre de nœuds soit comparable (haut: 108, bas : 95), la densité des rapports est très différent (degré moyen, haut: 2.4, bas: 12.8). En examinant de plus près on se rend comte que le premier groupe ne représente qu'un lieu d'échange de photos tandis que le deuxième implique des échanges intensifs, des réunions physiques, etc. Serait-il justifié de parler d'une "communauté » pour le deuxième cas ?

Nos recherches sur Flickr et delicious ont effectivement montré que les usages de ces systèmes peuvent aller d'une gestion purement personnelle de ses propres photos ou liens à leur emploi comme véritables plates-formes de publication. De manière analogue, les structures relationnelles qui se développent dans des groupes peuvent varier énormément (Fig. 1). Une étude quantitative d'un échantillon complet de Flickr, faite par des chercheurs français des Orange Labs (Prieur et al., 2008), confirme ce diagnostic d'une hétérogénéité fondamentale dans l'investissement des internautes, qui implique en grande partie des échanges éphémères et instables, une "coopération faible » dans les termes des auteurs.

Dans une situation où la masse n'est jamais qu'à un clic de souris, où les interactions de faible intensité nous mettent en permanence en contact avec l'autre, les entrées et sorties de la bulle doivent être filtrées et contrôlées de manière flexible. La métaphore de l'écume propose un instrument puissant pour penser ces modulations : une écume est formée d'inclusions de gaz dans un matériau liquide ou solide, un milieu, dont se composent les membranes qui séparent les bulles entre elles.

\subsection{Interface et filtre}

Étant donné que les bulles d'une écume sont co-isolées, les membranes qui les séparent sont à la fois des agents de séparation et de connexion, filtres et interfaces. Dans un environnement technique, les membranes sont faites d'une matière technique, c.-à-d. des formes et fonctions interactives qui servent aussi bien à aller vers les autres qu'à s'isoler d'eux. Commençons par quelques exemples de fonctions techniques, proposées par notre catalogue d'applications, qui visent effectivement la mise en relation avec d'autres internautes :

- À travers les blogrolls, les backlinks, les commentaires et les moteurs de recherche spécialisés, un blog peut rapidement prendre place dans une blogosphère qui le référence et, par conséquent, un auteur 
De la communauté à l'écume : quels concepts de sociabilité pour le « web social »?

peut se trouver entouré par un ensemble de personnes qui partagent son intérêt pour un sujet et souhaitent entrer en discussion avec lui. Les structures de sociabilité qui émergent portent la marque de la technicité qui rend leur existence possible. Pour trouver son audience, il faut être stratégiquement extroverti : publier, référencer et commenter le plus possible.

- Les folksonomies implémentées par des systèmes comme Flickr ou delicious sont utilisées pour créer des parcours de navigation qui impliquent en fait une double circulation: on navigue à la fois dans une base de contenus et dans une population d'usagers. À travers les différentes fonctionnalités de communication, le contact éphémère que représente le passage sur un profil d'utilisateur peut se développer (ou non) en une relation plus importante et stable.

- En proposant différentes fonctionnalités pour la création de groupes et la possibilité de chercher et naviguer dans la masse des membres, les sites de réseautage social proposent l'accès potentiel à tous les autres membres du système et fournissent des "facilitateurs " pour animer une éventuelle communication, p.ex. à travers le partage de certaines préférences culturelles ou la proximité sociale créée par un " ami » commun. Facebook p.ex. va donc proposer de nouveaux amis à ces membres.

- Les sites de rencontre servent évidemment à produire du contact. Ces systèmes brisent les limitations de l'espace physique et permettent à leurs membres de s'adresser à des très grosses populations de personnes. La possibilité d'effectuer de recherches complexes sur les profils est censée faciliter la recherche d'un partenaire "compatible » et réduire le "taux d'échec» des rencontres qui ne livrent pas les résultats souhaités. Certains sites comme p.ex. Parship travaillent avec des « indexes de compatibilité » et suggèrent automatiquement des partenaires potentiels " prometteurs".

On le remarque tout de suite : le web social introduit des nouveaux vecteurs de sociogenèse, des manières sociotechniques de production de liens sociaux. En partant des interfaces proposées par les membranes techniques, nous témoignons actuellement de l'émergence de nouvelles formes de prise de contact et de création de relation dont la médiation passe par la plasticité du substrat numérique. Or, pour paraphraser Heidegger, le contenu de la technique n'est jamais technique: les différentes plates-formes incorporent déjà des logiques sociales. II serait pourtant trop simpliste de regarder uniquement la dimension de mise en relation du web social. Les membranes numériques peuvent produire du contact, certes ; mais elles impliquent également du filtrage 
et de l'isolation contre la masse des autres. Une deuxième liste d'exemples doit donc compléter la première :

- Dans la plupart des logiciels de blog on trouve un système de gestion des droits d'utilisateur qui sert à limiter l'accès des internautes à certaines fonctions. On peut donc préciser qui peut lire le blog, qui peut le commenter et qui peut écrire des billets. Ainsi, un auteur peut décider d'utiliser son blog comme un bloc-notes purement personnel, inaccessible pour tout autre personne ou, au contraire, ouvrir toutes les fonctions à tout le monde. Selon le logiciel utilisé, le paramétrage de ce filtre peut être plus ou moins fin.

- Sur Flickr, l'accès aux photos et aux différentes fonctionnalités liées à ces photos (commenter, tagguer, géolocalisation) peut être finement contrôlé. La configuration se fait à travers quatre groupes: " seulement moi », " ma famille ", " mes amis ", " tout le monde ". Les contacts sont donc séparés en deux zones de proximité (famille et amis) ce qui permet une gestion encore plus subtile des permissions. De plus, ces permissions peuvent être appliquées différemment d'une image à l'autre.

- Facebook et d'autres sites de réseautage social intègrent aujourd'hui la possibilité d'ajuster très subtilement la visibilité de ses propres informations et activités. On peut, entre autres, gérer l'accès aux différents éléments de son profil, moduler les flux de notification et limiter l'accès par les moteurs de recherche interne et externe. La granularité de la perméabilité de la membrane protégeant un espace personnel est très fine et évolue en permanence. On peut largement fermer les portes d'entrée ou, au contraire, les ouvrir de manière radicale : l'application "honesty box» (boîte d'honnêteté) par exemple, disponible sur Facebook depuis juin 2007, permet aux usagers de recevoir les messages anonymes d'autres membres du système - la population ayant la permission étant toujours modulable.

- Sur les sites de rencontre, le paramétrage du contact potentiel va très loin. A travers les différentes catégories des profils (souvent très complets, allant jusqu'au test de personnalité), un usager peut non seulement chercher de manière très précise mais également déterminer par qui il peut être trouvé. II est donc possible de bloquer des personnes affichant (ou non) certaines caractéristiques comme le niveau d'étude ou une nationalité particulière. Certains services permettent de dévoiler son profil progressif au fil d'une conversation avec un autre membre, rendant la membrane plus transparente avec la croissance du niveau de confiance. Enfin, le site Parship fournit 
De la communauté à l'écume : quels concepts de sociabilité pour le « web social »?

une fonction qui empêche toute forme de contact futur avec un membre particulier. L'autre disparaît du système - "plonk*.

La notion de membrane renvoie ainsi à l'idée que le passage entre intérieur et extérieur peut être régulé en termes aussi bien de quantité que de qualité. Les caractéristiques spécifiques des fonctions " interface " et "filtre " d'une membrane dépendent très largement des spécifications du système en question. Les formes de sociabilité qui émergent dans ce système sont le résultat des dynamiques qui se développent entre les bulles à travers les médiations du milieu technique. Le terme " web social » prend donc un double sens: premièrement, il pointe vers la sociabilité de masse que l'on peut observer sur un ensemble de sites de grande popularité ; et deuxièmement, il indique que ces sites ouvrent des espaces sociaux conçus qui obéissent à des logiques techniques variables, des " architectures discursives " structurées autour de logiques d'activité particulières, qui ne fonctionnent pas nécessairement selon les principes organisateurs d'autres lieux de sociabilité.

La rapide évolution des capacités d'interfaçage et de filtrage des membranes répond en quelque sorte au succès de ce web social. Comme on parle d'une "surcharge d'information", on devrait peut-être parler d'une "surcharge sociale ». En brisant certaines limitations imposées par l'espace physique, les différents systèmes offrent un accès potentiel à de très grandes populations: Facebook affiche désormais plus de 300 millions de membres actifs ${ }^{10}$ et les plus grands sites de rencontre promettent un choix de millions de partenaires potentiels. Le succès de ces services dépend, au moins en partie, de leur capacité à répondre de manière efficace à la question de la " pertinence ", problématique centrale de la recherche d'information. Dans une situation où l'information abonde, le moteur de recherche est censé de faire le tri entre informations "pertinentes", qui répondent aux besoins de l'usager, et informations "non pertinentes", qui sont à écarter. Nous pensons que les membranes du Web social cherchent à faire, consciemment ou non, un tri similaire entre contact social "pertinent " et "non pertinent " à travers leur double fonction d'interface et de filtre. Les modalités interactives produisent un rapport de plus en plus sélectif à l'autre. La liberté de ces espaces est donc tout à fait relative et on peut se demander comment va évoluer la mixité sociale en ligne si la mise en contact de deux personnes dépend des test de compatibilité invisibles et universels fondés sur la comparaison de profils d'utilisateurs.

${ }^{10}$ http://www.facebook.com/press/info.php?statistics, consulté le 30 octobre 2009. 
Bernhard RIEDER

\section{Conclusion}

L'écume est finalement une métaphore pour la proto-sociabilité et par conséquent un outil pour penser la sociogenèse à partir d'un " individu empilé ». Du chaos des interactions éphémères émergent des relations plus stables et l'assemblage de ces relations produit des morphologies que nous cherchons à décrire par les concepts de communauté et de réseau social. Une tâche ou cause commune peut transformer l'écume temporairement en foule en l'orientant dans un même sens. Or, la métaphore de l'écume nous aide avant tout à théoriser cet individualisme de masse qui semble tellement marquant de notre temps et à penser la tension entre identité et relation comme locus de la production du social. A travers les idées de milieu et membrane, elle accorde une place importante à la technique dans les processus de sociabilité en ligne. Pour répondre à une de nos interrogations initiales, on peut dire que la plasticité du numérique apparaît sous une autre lumière : la création de systèmes de communication et d'échange donnant forme à cet objet malléable qu'est l'internet (et plus précisément le web) est non seulement une activité technique mais implique aussi effectivement une ingénierie du social. Les logiques à l'œuvre dans les différentes plates-formes ont été conçues et ce travail de conception est un geste politique.

Les outils et activités que l'on a résumé sous le signet « web social » ont suscité, comme les innovations le font souvent, des visions utopiques et dystopiques. Entre espoir de démocratisation et peur de déculturation (Keen, 2007) se dessine un ensemble de phénomènes dont l'analyse devrait se nuancer progressivement dans les années à venir. Pour l'instant, nous pensons qu'il est important de développer et peaufiner notre inventaire conceptuel et nos outils méthodologiques. Nous pensons effectivement que nous avons besoin d'une meilleure compréhension de cette multitude d'interactions éphémères qui restent au dessous du seuil de sociabilité qu'impliquent la "communauté » et le "réseau » et que nous avons tenté de rendre perceptible en utilisant la métaphore de l'écume. Pour l'instant, elle reste à l'état de métaphore et beaucoup de travail sera encore nécessaire pour la concrétiser en véritable concept. Or, son potentiel analytique et critique justifie un tel engagement.

\section{Références bibliographiques}

Boyd D., 2006, Friendster lost steam. Is MySpace just a fad? <http://www.danah.org/papers/FriendsterMySpaceEssay.html>, dernière consultation le 30 octobre 2009. 
De la communauté à l'écume : quels concepts de sociabilité pour le « web social »?

Bruns A., 2008, Blogs, Wikipedia, Second Life, and Beyond: From Production to Produsage, New York, Peter Lang.

Castells M., 1996-1998, The Information Age I-III, Cambridge MA, Oxford, Blackwell.

Canetti E., 1960, Masse und Macht, Frankfurt, Fischer.

Degenne A., M. Forsé, 2004, Les réseaux sociaux, Paris, Armand Colin.

Flichy P., 1999, « Internet ou la communauté scientifique idéale », Réseaux, vol.17, n97, p.77-120.

Georges F., A. Seilles, G. Artignan, B. Arnaud, M. Hascoët, N. Rodriguez, V. Dresp-Langley, J. Sallantin, 2009, "Sémiotique et visualisation de l'identité numérique: une étude comparée de Facebook et Myspace », dans I. Saleh, S. Leleu-Merviel, Y. Jeanneret, L. Massou, N. Bouhai (dir.), H2PTM'09 (Actes). Rétrospective et perspective : 1989 - 2009, Paris, Hermès-Lavoisier, pp.257-268.

Goldhaber M. H., 1997, The Attention Economy and the Net, First Monday, vol.2, $\mathrm{n}^{\circ} 4$, <http://www.firstmonday.org/issues/issue2_4/goldhaber/>, dernière consultation le 30 octobre 2009.

Granovetter M., 1973, "The Strength of Weak Ties », American Journal of Sociology, vol.78, $\mathrm{n}^{\circ} 6$, pp.1360-1380.

Jenkins H., 2006, Convergence Culture, New York, NYU Press.

Keen A., 2007, The Cult of the Amateur: How Today's Internet Is Killing Our Culture, New York, Doubleday.

Latham R., S. Sassen, 2005, Digital Formations : IT and New Architectures in the Global Realm, Princeton, Princeton University Press.

Leadbeater C., P. Miller, 2004, The Pro-Am Revolution, London, Demos.

Le Bon G., 1895, Psychologie des foules, Paris, Alcan.

Lovink G., 2006, « Digitale Nihilisten », Lettre International, n73, pp.94-99.

Prieur C., D. Cardon, J.-S. Beuscart, N. Pissard, P. Pons, 2008, The Stength of Weak cooperation: A Case Study on Flickr, arXiv:0802.2317v1, <http://arxiv.org/abs/0802.2317>, dernière consultation le 30 octobre 2009.

Rieder B., 2006, Métatechnologies et delegation. Pour un design orienté-société dans l'ère du Web 2.0, thèse de doctorat, Université Paris 8. 
Bernhard RIEDER

Rheingold H., 1993, The Virtual Community: Homesteading on the Electronic Frontier, New York, HarperPerennial.

Sack W., 2005, "Discourse Architecture and Very Large-scale Conversation », dans R. Latham, S. S. Sassen, (dir.), Digital Formations : IT and New Architectures in the Global Realm, Princeton, Princeton University Press, pp.242-282.

Saltzer J. H., D. P. Reed, D. D. Clark, 1984, « End-to-end arguments in system design », ACM Transactions on Computer Systems, vol.2, n 4, pp.277-288.

Sloterdijk P., 1998-2004, Sphären I-III, Frankfurt/M., Suhrkamp.

Surowiecki J., 2004, The Wisdom of Crowds, New York, Doubleday.

Tönnies F., 1887, Gemeinschaft und Gesellschaft, Leipzig, Fues' Verlag.

Turing A. M., 1948, Intelligent Machinery, National Physical Laboratory Report, <http://www.alanturing.net/turing_archive/archive////32/L32-001.html>, dernière consultation le 30 octobre 2009

Watts D. J., 2005, « The 'New' Science of Networks », Annual Review of Sociology, vol.30, $\mathrm{n}^{\circ} 1$, pp.243-270. 